\title{
Visual Monitoring of Complex Algorithms
}

\author{
Michele Invernizzi ${ }^{1}$, Michele Mauri ${ }^{1}$ and Paolo Ciuccarelli ${ }^{1}$ \\ Politecnico di Milano, Department of Design, \\ Via Durando 38/A - 20158 Milano, Italy \\ \{michele.invernizzi, michele.mauri, paolo.ciuccarelli\}@polimi.it
}

\begin{abstract}
Purchases, conversations, access to information, music and movies: more and more of our online life is mediated by complex algorithms that are designed to make the experience of the Web customized and more "personal". These algorithms can process an amount of heterogeneous data that would take enormous resources for the human mind to cope with, and find valuable patterns in it. Their use is not limited to our online experience as similar algorithms have been also implemented, for example, to inform policymakers: suggesting where to deploy police forces around the urban context, assessing criminality risk scores of offenders, or allocating high school students to the most suited school.

While the consequences of the decisions made by algorithms have a great impact on people's lives, the way they are built and designed makes them de facto "black boxes": a series of legal and technical barriers prevents from accessing and understanding how a certain input influences a given output. Overseeing their decision processes becomes then of the utmost importance.

This paper argues that visualizations can become a powerful tool to monitor algorithms and make their complexity accessible and usable by visually showing the relation between the inputs and the outputs in a manner that mimics an observational study approach. The paper analyzes a case study developed as an experiment to test opportunities and criticalities in using visualization to represent the presence and the activity of algorithms.

This represents a shift from the main purpose of visualizations and Data Visualization in general: since its strong suit is to support human decisionmaking processes by transforming data into knowledge, the substitution of people by machines in this activity seems to make visualizations obsolete. A computer doesn't need to "see" the data to make a decision - or at least not in the same way as people do - no matter how multidimensional and heterogeneous the data is. With the diffusion of algorithms, the need to inspect their accountability and performance will simply move visualizations at a later stage. From a decision-making tool visualization becomes a monitoring and awareness tool.
\end{abstract}

Keywords: Data Visualization / Algorithmic Accountability / Visualizing complexity 


\section{Introduction}

It has become almost formulaic to say: "We now live in a hyperconnected, online world", and yet it still is a phrase that is worth saying because every year, or better every day, a bigger portion of our daily interactions with entities different than ourselves could potentially be moved entirely online. Supplies are bought on Amazon, vacations are booked on Airbnb, news are read on Facebook, etc. For every need and desire there is a platform for that. Moreover, the shift to the digital has been so powerful also because most of these interactions are tailored just for us, to the point where no person sees a given website in the same way and no website is seen in the same way twice. This has become possible since every action performed online leaves digital traces, that can be collected, processed and later disseminated [1] as a personalized service by firms whose central core is built around this ability of analyzing data [2].

Managing such massive inflow of multidimensional and heterogeneous data would be impossible without the help of powerful algorithms that can cope with and find valuable patterns in the complexity of the data. These algorithms are designed to be dynamic [3], in the sense that they adapt to the ever-changing data, and self-taught as they make decisions without the need by their programmers to hardcode all the possibilities when facing these decisions [4]. Fed by the data we produce daily they mediate, invisible and undetected [5], most of our Web interactions: search engine results, photos and texts on social networks, movies or products suggestions are all managed by a set of computer programs that are most commonly known as machinelearning algorithms.

The use of these tools is not only confined to the online realm, as similar algorithms are beginning to be employed to inform policymakers in the public sector or affect us in our offline life. Examples are predictive policing algorithms that are used to deploy effectively police forces around the city ${ }^{1}$, assessment algorithms that are used by judges to inform their decision by providing a criminality risk score to the offender ${ }^{2}$, or data processing algorithms that control self-driving cars. Especially when we consider the latter examples, a set of questions arise: how do we evaluate the decision of the algorithm? How can we understand the logics followed by the algorithm in choosing a particular solution?

As soon as we start to think in terms of algorithmic accountability [3], what makes these computer programs so powerful and flexible turns out to be also their greatest pitfall. In fact, in order to make decisions based on provided data, a machine-learning algorithm creates an internal layer that is not understandable even by the programmers that wrote that algorithm [6]. So, even if the data and the code written by the engineers are comprehensible, the way the algorithm "learns" from the data and later makes decisions is not: this combination of algorithm and data [7] is where its complexity, and opacity, resides. Moreover, all the code behind the proprietary

\footnotetext{
${ }^{1}$ http://www.predpol.com

${ }^{2}$ http://www.equivant.com
} 
software is jealously protected by intellectual property patents and away from possible public scrutiny. It thus becomes a black box [8] that is hard to evaluate and tools are needed to make sure that there is control or at least transparency over risks of fault or, worse, discrimination done by the algorithm.

\section{Exposing algorithms through visualization}

We argue that one of the tools that could be used to expose these algorithms and engage publics in a fruitful discussion of the issue comes from information design, namely information visualization. With it we are able to trace the origins and presence of an issue, in this case the presence of an algorithm, and communicate it, make it known [9].

In this paper we present a case study where visualization was aimed at investigating the News Feed algorithm of Facebook. This content curation algorithm is particularly interesting because its use is widespread and relevant, as it decides what should be shown to 2.13 billion monthly active Facebook users ${ }^{3}$; at the same time its presence is invisible, as many users are not aware of its existence at all [10].

The project was the result of a collaboration with a group of digital activists, led by Claudio Agosti, who are developing tools with the goal of increasing transparency and awareness behind personalization algorithms. They developed a browser extension called facebook.tracking.exposed ${ }^{4}$ that collects data about the posts seen whenever using Facebook: namely the time of creation of the post, its type and its order of appearance in the user's feed. In order to make this data readable and comparable we created an interactive visualization trough which users can see their interaction with Facebook from a different perspective, exposing how the News Feed algorithm rearrange results compared to their temporal flow. Since it's technically and legally impossible to reverse-engineer the algorithm or understand its internal structure, in this case study information visualization plays a key role in revealing the algorithm presence and highlight its outputs, thus creating a way to open the black box [11].

\footnotetext{
${ }^{3}$ Data taken from https://newsroom.fb.com/company-info, updated April $4{ }^{\text {th }} 2018$

${ }^{4}$ https://facebook.tracking.exposed. At the time of the collaboration the project was still in its alpha phase and it is now much evolved since then.
} 

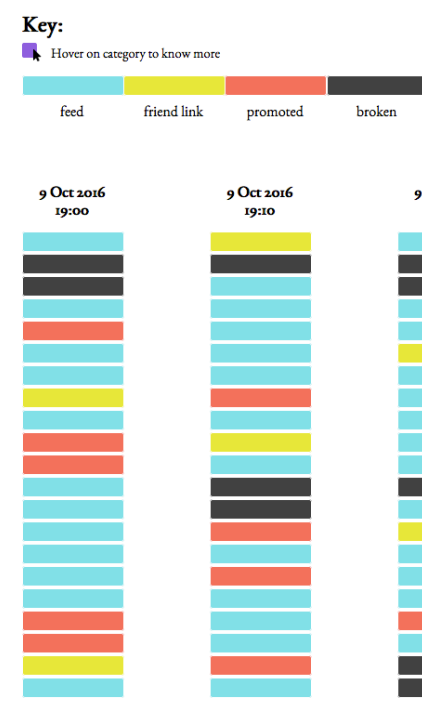
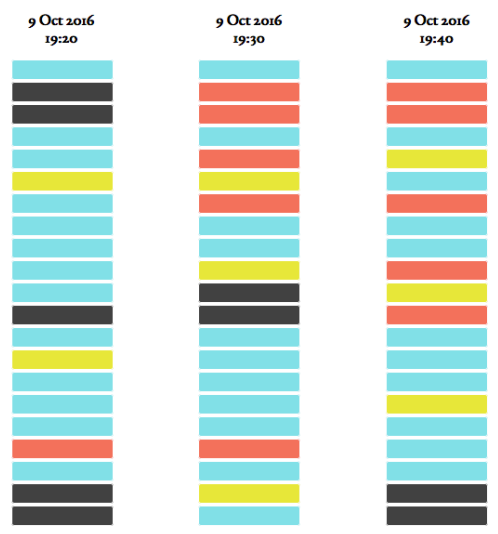

Sort by type

Sort by actual creation time

Show allinfo

Fig. 1. Screenshot of the interface showing the posts grouped in columns (representing the moments in time when the user went on Facebook).

\section{Interface and use case}

After installing the extension, every time the user opens Facebook, the order and type of posts is recorded. In any moment the user can insert her Facebook Id in the interface in order to load the visualization with her own data. The interface presents every visit to the social network as a column, sorted in chronological order. Each column is composed by seen posts, color coded by the type of post (Fig. 1). The user could then sort the posts based on different criteria, in a way overwriting the curation of the algorithm: they can be sorted by type or by actual creation time. Hovering on a post would highlight it through time, letting the user see the relevance given to it by the algorithm in different moments (Fig. 2). By letting the users play with the order of Facebook posts, we are both creating awareness of the existence of the algorithm as well as engaging them in thinking about the consequences of the curation mechanism. Taking as example Fig. 1, we can see that the user has been on Facebook on October $9^{\text {th }} 2016$ six times, once every ten minutes. Leaving aside posts colored in black ${ }^{5}$, we can notice that there are quite a lot of promoted posts (advertising paid by companies), especially in the last three columns where they are even among the first posts to be shown. Another thing we could see is that friend links, meaning posts made by friends of friends that Facebook thinks could be relevant to us, are sporadic but quite evenly spread out.

\footnotetext{
${ }^{5}$ These would be broken posts, or posts that the browser extension was not able to retrieve.
} 

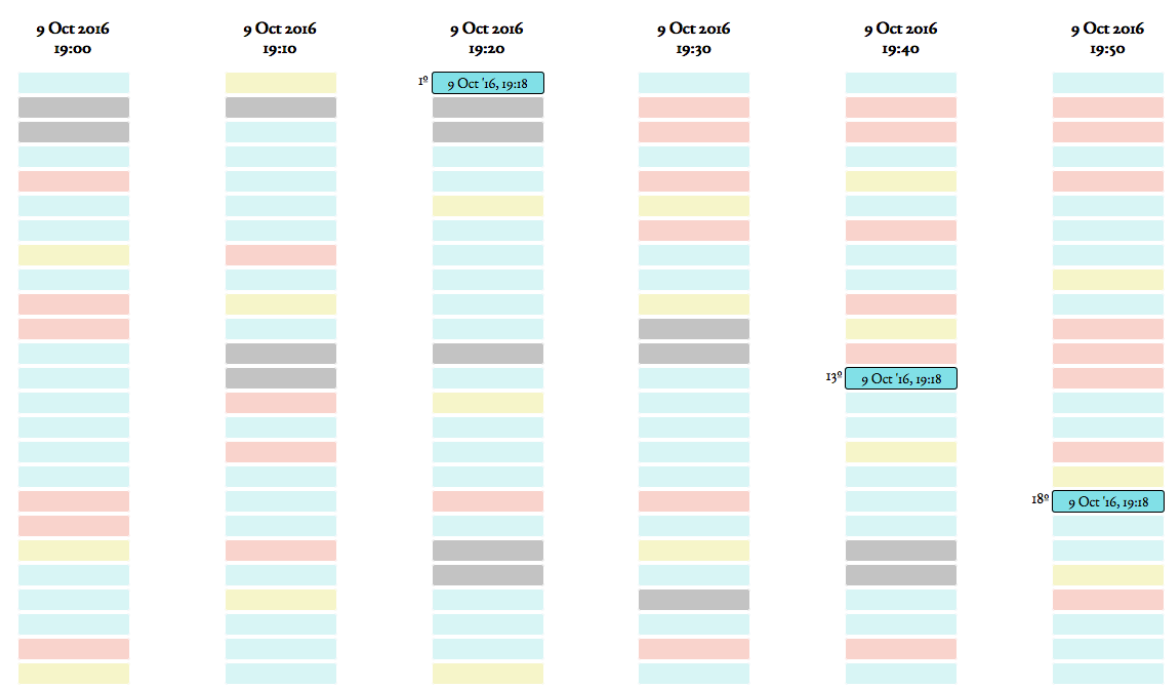

Fig. 2. Screenshot of the interface showing the same post highlighted in different columns.

\section{Discussion}

With this case study, we've seen how we can use information visualization to expose an invisible algorithm. During the unfolding of the project, a series of limitations and prompts for future development have emerged and are worth discussing.

First of all, it's important to keep in mind that this case study had some ethical implications: by using Facebook data we were not complying with its Terms of Service, even more since we were using data collected automatically by a program. At this point we had to make a calculation of the trade-offs between this violation and the benefits of studying a potential harmful discrimination. That said, the other relevant limitation is in terms of scale of the data. What it means is that our ability of exposing the algorithm is of course strictly related to how much information we can gather about it. If we could have accessed not only the order of the posts seen by the user and their type but also for example, the total number of friends that the user had, we could have calculated how many friends the algorithm was deciding to show, or which friend was considered the most relevant by it. Overall though, this first attempt opened the way to a series of future experiments where we will also need to set up an evaluation process. At the same time, the project revealed an interesting new way of thinking about the purpose of visualization. While we think of it as a good way to make sense of complex data to inform the process of decision-making, this purpose would seem at first glance to lose meaning the moment we start to delegate every decision to computer programs: they now decide what is more relevant, more coherent, more suited to our needs. Visualization would nevertheless still be useful, moving from a decision-making to a monitoring and awareness tool. 


\section{References}

[1] D. J. Solove, "A taxonomy of privacy," U. Pa. L. Rev., vol. 154, p. 477, 2005.

[2] N. Srnicek, Platform capitalism, John Wiley \& Sons, 2017

[3] N. Diakopoulos, "Algorithmic accountability: Journalistic investigation of computational power structures," Digital Journalism, vol. 3(3), pp. 398-415, 2015.

[4] J. Burrell, "How the machine 'thinks': Understanding opacity in machine learning algorithms," Big Data |\& Society, vol. 3(1), 2016.

[5] K. Hamilton, K. Karahalios, C. Sandvig and M. Eslami, "A path to understanding the effects of algorithm awareness," in CHI'14 Extended Abstracts on Human Factors in Computing Systems, 2014.

[6] T. Gillespie, "The relevance of algorithms," Media technologies: Essays on communication, materiality, and society, p. 167, 2014.

[7] C. Sandvig, K. Hamilton, K. Karahalios and C. Langbort, "Auditing algorithms: Research methods for detecting discrimination on internet platforms," Data and discrimination: converting critical concerns into productive inquiry, pp. 1-23, 2014.

[8] F. Pasquale, The black box society: The secret algorithms that control money and information, Harvard University Press, 2015.

[9] C. DiSalvo, "Design and the Construction of Publics," Design issues, vol. 25, no. 1, pp. 4863, 2009.

[10] M. Eslami, A. Rickman, K. Vaccaro, A. Aleyasen, A. Vuong, K. Karahalios, K. Hamilton and C. Sandvig, "I always assumed that I wasn't really that close to [her]: Reasoning about Invisible Algorithms in News Feeds," in Proceedings of the 33rd annual ACM conference on human factors in computing systems, 2015.

[11] B. Latour, Science in action: How to follow scientists and engineers through society, Harvard university press, 1987. 